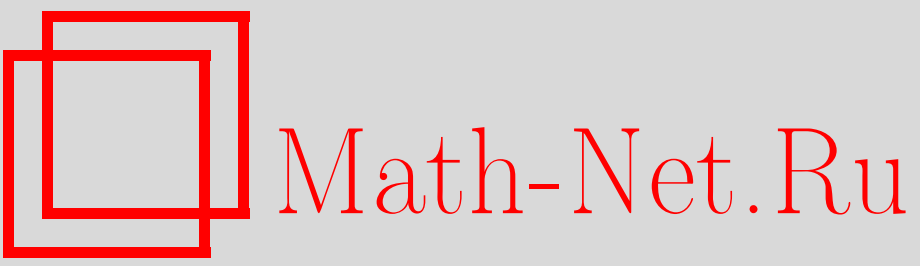

Д. А. Деревнин, А. Д. Медных, О формуле объема гиперболического тетраэдра, УМН, 2005, том 60, выпуск 2, 159-160

DOI: https://doi.org/10.4213/rm1414

Использование Общероссийского математического портала Math-Net.Ru подразумевает, что вы прочитали и согласны с пользовательским соглашением

http://www.mathnet.ru/rus/agreement

Параметры загрузки:

IP : 107.22 .136 .117

26 апреля 2023 г., 12:24:09 


\title{
О ФОРМУЛЕ ОБЪЕМА ГИПЕРБОЛИЧЕСКОГО ТЕТРАЭДРА
}

\author{
Д. А. ДЕРевнин, А. Д. МЕДных
}

Введение. Вычисление объема многогранника в трехмерном пространстве является очень старой и трудной проблемой. Первый известный резултат в этом направлении принадлежит Тарталье (1494 г.), которьй нашел объем евклидова тетраэдра. Сейчас этот результат известен как формула Кэли-Менгера. Недавно в [1] и [2] было показано, что объем любого евклидова многогранника является корнем алгебраического уравнения, коэффициенты которого зависят от длин ребер и полностью определяются комбинаторным типом многогранника.

В гиперболическом и сферическом пространствах ситуация более сложная. Формула объема для биортогонального тетраэдра в этих пространствах известна со времен Лобачевского и Шлефли [3]. Объем правильного тетраэдра в гиперболическом пространстве найден в [4]. Случай гиперболического тетраэдра с несколькими идеальными вершинами исследован в [3]. Формула объема для произвольного тетраэдра оставалась долгое время неизвестной. Общий алгоритм для нахождения такой формулы намечен в [5]. Полное решение проблемы получено совсем недавно несколькими авторами [6]-[8]. Все результаты представлены в виде комбинации 16 дилогарифмических функций или функций Лобачевского, зависящих от двугранных углов тетраэдра и некоторых вспомогательных параметров, являющихся корнями достаточно сложного квадратного уравнения с комплексньми коэффициентами. Геометрический смысл формулы Мураками-Яно [7] разъяснен в [9] с точки зрения так назьваемой симметрии Редже. Замечателшное изложение этих идей и полное геометрическое доказательство формулы дано в работе [10].

Заметим, что формула для объема существенно упрощается в случае симметрического тетраэдра, т.е. тетраэдра, противоположные двугранные углы которого равны. Впервые это было обнаружено Милнором [11] для идеального тетраэдра. Позже в [12] было показано, что достаточно простая формула объема существует и для произвольного симметрического тетраэдра с собственными вершинами.

В настоящей работе предложена элементарная интегральная формула для объема гиперболического тетраэдра. При этом, объем выражается через простые геометрические характеристики, зависящие от двугранных углов тетраэдра. Формула удобна для реализации на компьютере, а резулттаты работ [6]-[8] получаются из нее как следствия.

Объем гиперболического тетраэдра. Пусть $T(A, B, C, D, E, F)$ - компактный гиперболический тетраэдр с двугранньми углами $A, B, C, D, E, F$, выбранными таким образом, что углы $A, B, C$ лежат при одной вершине, а углы $D, E, F$ противолежат углам $A, B, C$ соответственно.

Tеорема 1. Обгем гиперболического тетраәдра $T=T(A, B, C, D, E, F)$ равен

$$
\operatorname{Vol}(T)=-\frac{1}{4} \int_{z_{1}}^{z_{2}} \log \frac{\cos \frac{A+B+C+z}{2} \cos \frac{A+E+F+z}{2} \cos \frac{B+D+F+z}{2} \cos \frac{C+D+E+z}{2}}{\sin \frac{A+B+D+E+z}{2} \sin \frac{A+C+D+F+z}{2} \sin \frac{B+C+E+F+z}{2} \sin \frac{z}{2}} d z,
$$

где $z_{1}$ и $z_{2}$ - корни подынтегрального выражения, определяемые формулами

где

$$
z_{1}=\operatorname{arctg} \frac{k_{2}}{k_{1}}-\operatorname{arctg} \frac{k_{4}}{k_{3}}, \quad z_{2}=\operatorname{arctg} \frac{k_{2}}{k_{1}}+\operatorname{arctg} \frac{k_{4}}{k_{3}},
$$

$$
\begin{aligned}
k_{1}=- & (\cos S+\cos (A+D)+\cos (B+E)+\cos (C+F)+\cos (D+E+F) \\
& \quad+\cos (D+B+C)+\cos (A+E+C)+\cos (A+B+F)), \\
k_{2}= & \sin S+\sin (A+D)+\sin (B+E)+\sin (C+F)+\sin (D+E+F)+\sin (D+B+C) \\
& \quad+\sin (A+E+C)+\sin (A+B+F), \\
k_{3}= & 2(\sin A \sin D+\sin B \sin E+\sin C \sin F), \\
k_{4}= & \sqrt{k_{1}^{2}+k_{2}^{2}-k_{3}^{2}}, \\
u S= & A+B+C+D+E+F .
\end{aligned}
$$

Работа выполнена при поддержке INTAS (грант 03-51-3663) и РФФИ (гранты 03-01-00104, 02-01-22004a). 
ЗАмечание 1. Суммы двугранных углов $V_{1}=A+B+C, V_{2}=A+E+F, V_{3}=B+D+F$, $V_{4}=C+D+E$ в числителе формулы объема и $H_{1}=A+B+D+E, H_{2}=A+C+D+F$, $H_{3}=B+C+E+F$ в ее знаменателе имеют простой геометрический смысл. А именно, $V_{1}, V_{2}$, $V_{3}, V_{4}$ являются суммами двугранных углов при вершинах тетраэдра $T$, в то время как $H_{1}, H_{2}$ и $H_{3}$ - суммы углов вдоль гамильтоновых циклов $T$.

ЗАмечАниЕ 2. Пределы интегрирования $z_{1}$ и $z_{2}$ удовлетворяют уравнению $k_{1} \cos z+$ $k_{2} \sin z=k_{3}$, а $k_{4}^{2}=-4 \operatorname{det}(G)$, где $G$ - матрица Грама тетраэдра $T$. Геометрический смысл величин $z_{1}$ и $z_{2}$ может быть извлечен из результатов работы [10]. Они возникают как параметры разбиения идеального октаэдра на четыре идеальных тетраэдра с общим ребром. Октаэдр канонически определяется тетраэдром $T$, а его двугранные углы являются линейными комбинациями двугранных углов $T$.

Рассмотрим дилогарифмическую функцию, определенную следующим интегралом:

$$
\operatorname{Li}_{2}(x)=-\int_{0}^{x} \frac{\log (1-t)}{t} d t,
$$

где $x \in \mathbb{C} \backslash[1, \infty)$, а непрерывная ветвь $\log \xi=\log |\xi|+i \arg \xi$ определяется условиями $-\pi<$ $\arg \xi<\pi$. Положим также $l(z)=\operatorname{Li}_{2}\left(e^{i z}\right)$.

Непосредственным следствием теоремы 1 является следующий результат, полученньй ранее в [7] и [8].

СлеДСтвиЕ 1. Гиперболический обгем тетраэдра $T$ равен $\operatorname{Im}\left(U\left(z_{1}, T\right)-U\left(z_{2}, T\right)\right) / 2$, əдe

$$
\begin{gathered}
U(z, T)=(l(z)+l(A+B+D+E+z)+l(A+C+D+F+z)+l(B+C+E+F+z) \\
-l(\pi+A+B+C+z)-l(\pi+A+E+F+z) \\
-l(\pi+B+D+F+z)-l(\pi+C+D+E+z)) / 2 .
\end{gathered}
$$

Для упрощения формулы Мураками-Яно заметим, что $\operatorname{Im}(l(z))=\operatorname{Im}\left(\operatorname{Li}_{2}\left(e^{i z}\right)\right)=2 \Lambda(z / 2)$, где $\Lambda(z)$ - функция Лобачевского, определенная интегралом

$$
\Lambda(z)=-\int_{0}^{z} \log |2 \sin t| d t .
$$

Таким образом, объем тетраэдра представляется в виде линейной комбинации шестнадщати функций Лобачевского.

\section{СПИСОК ЛИТЕРАТУРЫ}

[1] И.Х. Сабитов // Вестник Моск. ун-та. Сер. 1. Матем., мех. 1996. №6. С. 89-91. [2] R. Connelly, I. Sabitov, A. Walz // Beiträge Algebra Geom. 1997. V. 38. № 1. Р. 1-10. [3] Д. В. Алексеевский, Э. Б. Винберг, А. С. Солодовников, О. В. Шварцман. Геометрия-2 // Итоги науки и техники. Сер. Соврем. проблемы матем. Фундам. напр. т. 29. М.: ВИНИТИ, 1988. [4] G. J. Martin // Math. Chronicle. 1991. V. 20. P. 127-147. [5] W.-Y. Hsiang // Quart. J. Math. Oxford Ser. (2). 1988. V. 39. № 156. P. 463-468. [6] Yu. Cho, H. Kim // Discrete Comput. Geom. 1999. V. 22. №3. P. 347-366. [7] J. Murakami, M. Yano. On the volume of a hyperbolic and spherical tetrahedron // Preprint, 2002; http://www.f.waseda.ac.jp/murakami/papers/tetrahedronrev3.pdf. [8] A. Ushijima. A volume formula for generalized hyperbolic tetrahedra // Preprint, 2003; arXiv: math.GT/0309216. [9] G. Leibon. The symmetries of hyperbolic volume // Preprint, 2002. [10] Y. Mohanty // Algebr. Geom. Topol. 2003. V. 3. P. 1-31. [11] J. Milnor // Bull. Amer. Math. Soc. 1982. V. 6. № 1. Р. 9-24. [12] Д. А. Деревнин, А. Д. Медных, М. Г. Пашкевич // Сиб. матем. журн. 2004. V. 45. № 5. P. 1022-1031.

Институт математики им. С. Л. Соболева СО РАН

E-mail: derevnin@mail.ru; mednykh@math.nsc.ru
Представлено Э. Б. Винбергом Принято редколлегией 25.01.2005 